\title{
A Neurocognitive Assessment of a Patient with Body Integrity Identity
} Disorder

\author{
Philip J Sumner ${ }^{1}$, David J Castle ${ }^{2}$, Ryan A Kaplan ${ }^{3,4}$ and Susan L Rossell ${ }^{1,2,3,4^{*}}$ \\ ${ }^{1}$ Brain and Psychological Sciences Research Centre, Swinburne University of Technology, Melbourne, Victoria, Australia \\ ${ }^{2}$ Department of Psychiatry, St. Vincent's Hospital and University of Melbourne, Melbourne, Victoria, Australia \\ ${ }^{3}$ School of Psychology \& Psychiatry, Monash University, Melbourne, Victoria, Australia \\ ${ }^{4}$ Monash Alfred Psychiatry Research Centre, The Alfred and Central Clinical School, Monash University, Melbourne, Victoria, Australia
}

\section{Abstract}

Objective: Body integrity identity disorder is characterized by a desire to obtain a physical disability, often in the form of amputation. To date, we are aware of no comprehensive investigations of neurocognition in body integrity identity disorder.

Method: We report a comprehensive neurocognitive workup of an individual (Mr A), who presented with a longstanding and intense desire to amputate his left leg.

Results: In comparison to four demographically matched healthy controls, Mr A exhibited impairment in working memory, with some evidence of additional difficulties with long-term memory.

Conclusions: His neurocognitive weaknesses are inline with altered functioning within the superior parietal lobule, which has been implicated in the pathophysiology of body integrity identity disorder.

\section{Publication History:}

Received: May 03, 2015

Accepted: July 01, 2015

Published: July 03, 2015

\section{Keywords:}

Body Integrity Identity Disorder, Working memory, Attention, Superior parietal lobule

\section{Introduction}

Body integrity identity disorder is characterized by a desire to obtain what most would consider a physical disability, often in the form of limb amputation [1-6]. This desire is primarily motivated by a disparity between the individual's biological bodily configuration and their subjective experience of how their body is 'supposed' to be, despite sustained awareness of the biological integrity of their own body. The disorder is chronic, with a typical onset in childhood. Yet presentation is often in middle adulthood, with many sufferers maintaining a degree of secrecy surrounding their desires such that loved ones or therapists may not be aware of the presence of the condition. Alarmingly, body integrity identity disorder can lead to severe self-inflicted injuries directed towards undesired limbs.

Research to date has predominantly been concerned with capturing the general features of this poorly understood disorder. Concordance amongst these studies suggests that body integrity identity disorder most frequently afflicts males, and typically involves a desire to amputate the leg above the knee $[1,5,6]$. Individuals report a strong preoccupation with their symptoms and there is often comorbidity, including depression and anxiety $[1,2,3,5,7]$.

One of the remarkable characteristics of body integrity identity disorder is that those with the disorder exhibit preserved insight into the abnormality of their desire for amputation; this sets it apart from psychosis [4]. Furthermore, the disorder has been documented in highly educated individuals $[1,3,5]$ and normal neuropsychological performance in body integrity identity disorder has been noted [7]. As such, body integrity identity disorder symptomatology seems to revolve around the desire for body modification in a fairly specific manner, and so those with the disorder might otherwise be regarded as cognitively intact. However, there has not yet been any direct empirical assessment of this assumption. First [3] reported on the largest sample of potential body integrity identity disorder patients in the literature. His sample consisted of self-identified individuals who had a desire to have an amputation, each completing telephone interview. Almost half of this group reported impairments that included difficulties in concentration and focus with respect to their occupations and leisure activities. This implies that cognitive disruption might be apparent, although no explicit cognitive complaints were documented in these cases. Nevertheless, the possibility of perturbed cognition in body integrity identity disorder, specifically hyper attention towards undesired body parts, has been previously proposed [8].

There are some emerging neurobiological investigations in small samples with body integrity identity disorder. McGeoch et al. [9] found that the right superior parietal lobule of body integrity identity disorder patients specifically exhibited attenuated activity in response to tactile stimulation within an area of desired amputation. More recently, Hilti et al. [7] found evidence of reduced cortical thickness in the right superior parietal lobule of 13 participants with body integrity identity disorderrelative to 13 matched controls using structural magnetic resonance imaging. Furthermore, reduced cortical surface area was found in the primary and secondary somatosensory cortices, inferior parietal lobule, and anterior insular cortex of the right hemisphere. In the left hemisphere, larger cortical surface areas were associated with the secondary somatosensory cortex and inferior parietal lobule. Based on these findings, the authors proposed that, for people with body integrity identity disorder, a failing of the right superior parietal lobule to represent a particular limb results in increased arousal. Consequently, individuals developed a sense of aversion and a desire to amputate the affected body part.

There is agreement more generally that the superior parietal lobule is involved in a number of cognitive constructs and processes such as attention, conscious perception, episodic memory retrieval, and working memory [10]. It is noteworthy, then, that the function of informational integration and activity of the superior parietal lobule is considered to be important in cognition broadly, in addition to body representation.

In sum, there have been minimal attempts to investigate the *Corresponding Author: Prof. Susan Rossell, Swinburne University, Burwood Rd, Hawthorn, Melbourne, Australia, VIC 3122, Tel: +61 03-9214-8173; E-mail: srossell@srossell.com

Citation: Sumner PJ, Castle DJ, Kaplan RA, Rossell SL (2015) A Neurocognitive Assessment of a Patient with Body Integrity Identity Disorder. Int J Clin Case Stud 1: 107. doi: http://dx.doi.org/10.15344/2455-2356/2015/107

Copyright: (c) 2015 Summer et al. This is an open-access article distributed under the terms of the Creative Commons Attribution License, which permits unrestricted use, distribution, and reproduction in any medium, provided the original author and source are credited. 
neuropsychology of body integrity identity disorder [1]. The primary aim of the current study was to address this gap by administering an extensive neuropsychological battery to an individual with body integrity identity disorder as well as to demographically matched controls. It was hypothesized that the individual with the disorder would perform less accurately on tests requiring attention and working memory in comparison to relevant norms as well as the matched controls, given the potential involvement of the superior parietal lobule and body representation both in these tasks and in body integrity identity disorder.

\section{Method}

\section{Participants}

Case background: $\mathrm{Mr}$ A was a 31 year old male of a South American background, working as a self-employed music teacher. He was highly educated, having successfully completed a total of 17 years of education. His highest level of qualification was a Postgraduate Diploma in Music, which was completed in Australia. However, during the period in which this study was conducted, he had decided to return to university to complete a further degree necessary to become a primary school teacher. Notably, Spanish was Mr A's primary language, his fluent and articulate grasp of English notwithstanding. He wrote with his right hand.

At the age of 30, Mr A presented with a desire to obtain an aboveknee amputation of his left leg, despite acknowledging that such an operation would result in substantial impairments in his daily functioning. He did not view his left leg as deformed, misshapen, or otherwise abnormal, yet believed that he would be better off if it was removed. He was referred to a psychiatrist by his general practitioner and was subsequently diagnosed with body integrity identity disorder. At the time of testing, he was not taking any medications or receiving any treatment. He reported never having a head injury in the past; and his MRI scan was unremarkable as reported on by a neuroradiologist blind to his diagnosis

Controls: Despite the normative data associated with many neuropsychological tests, four matched controls were additionally employed in the current study. Given Mr A's musical background, a central concern was to recruit controls with similar levels of musicianship. Increased superior parietal lobule grey matter volumes thought to reflect enhanced sensorimotor integration [11] have been documented in musicians. Thus, controlling for these influences of musical training was deemed of importance, and all four controls reported proficiency in playing an instrument.

The average age of the controls was 28.25 years old $(\mathrm{SD}=4.27)$, their mean years of education was $17.63(\mathrm{SD}=3.04)$, and were right handed males (confirmed by the Edinburgh Handedness Inventory, [12]). There was no history of head injuries in any of the participants and none were taking any psychotropic medication.

\section{Materials}

Clinical assessment: To ensure that all participants were free from DSM-IV Axis 1 psychiatric diagnoses all were administered the MINI International Neuropsychiatric Interview $-5^{\text {th }}$ Edition (MINI-5)[13] In addition, to ensure that mood was not a confounding factor, all participants completed the Depression, Anxiety, and Stress Scale - 21 item version (DASS-21); a self-report index with separate sub-scales for ratings of depression, anxiety, and stress [14]. Scores for each sub-scale range from 0 to 21 , with higher scores indicating greater symptom severity.

\section{Neurocognitive Tests}

The Wechsler Adult Intelligence Scale: Fourth Edition (WAIS-IV). The WAIS-IV is a battery of subtests designed to evaluate adult intellectual abilities [15]. Age-corrected measures of both global intellectual functioning (i.e. the Full Scale Intelligence Quotient, FSIQ; and the General Ability Index, GAI) and functioning within four specific cognitive domains (i.e. Verbal Comprehension, Perceptual Reasoning, Working Memory, and Processing Speed) are provided. These evaluations are primarily determined from 10 core subtests (Vocabulary, Information, Similarities, Digit Span, Arithmetic, Block Design, Matrix Reasoning, Visual Puzzles, Coding, and Symbol Search). Scaled scores can be determined in relation to age-appropriate norms. Subtest scaled scores range from 1 to 19 , with a mean of 10 and a standard deviation of 3 , and with higher scores conveying better performance.

The Wechsler Memory Scale: Fourth Edition (WMS-IV).The WMSIV is a battery designed to evaluate various aspects of memory functioning [16]. In the current study, the Logical Memory I, Logical Memory II, and Logical Memory Recognition were utilized to assess verbal memory encoding and retrieval; and Visual Reproduction I, Visual Reproduction II, and Visual Reproduction Recognition were utilized to assess visuospatial memory processes. Further, Spatial Addition and Symbol Span were administered, which allowed for the calculation of Visual Working Memory Index scores.

The Visual Object and Space Perception Battery (VOSP): The VOSP is a battery of eight tests that are designed to assess object and space perception [17]. Namely, Incomplete Letters, Silhouettes, Object Decision, and Progressive Silhouettes assess object perception, and Dot Counting, Position Discrimination, Number Location, and Cube Analysis assess space perception.

Hopkins Verbal Learning Test: Revised (HVLT-R).The HVLT-R contains 12 nouns, four words each from one of three semantic categories (e.g., precious gems, animals, places to live), to be learned over the course of three learning trials. Approximately 20-25 min later, a delayed recall trial and a recognition trial are completed. The stratified normative data associated with the MCCB was used in the current study, which allowed for the calculation of $\mathrm{T}$ scores for the composite recall index from within age-, gender-, and educationappropriate strata [18]. For all of the other indices (i.e. the three individual learning trial scores, the recognition trial score and the delayed recall trial score) percentiles we calculated from an alternate normative data set, which was stratified by age [19].

The Hayling Sentence Completion Test and the Brixton Test: The Hayling and Brixton Tests are designed to measure executive functioning [20]. Two sections comprise the Hayling, both of which consist of 15 sentences with the last word omitted from each. In the first section, the examiner reads the sentences aloud and the examinee is required to provide a suitable last word to complete each one as quickly as possible. The sum of response latencies (initiation speed) is obtained and converted into a scaled score based on normative data. In the second section, the participant is required to provide a word that is semantically unconnected to the sentence. Two types of errors 
Citation: Sumner PJ, Castle DJ, Kaplan RA, Rossell SL (2015) A Neurocognitive Assessment of a Patient with Body Integrity Identity Disorder. Int J Clin Case Stud 1: 107. doi: http://dx.doi.org/10.15344/2455-2356/2015/107

Page 3 of 9

are possible depending on whether the provided word is connected or partially connected to the sentence. The scaled score reflecting total response latency is added to the scaled score reflecting total errors to indicate the examinee's capacity to suppress responses. The scaled scores from both sections are then summed and converted to provide an overall scaled score. All of the scaled scores produced range from 1 to 10 , with a mean of 6 , where higher scores denote better performance.

The Brixton involves the serial presentation of an array of 10 circles organized into two rows of five circles each [20]. On each page, one of the circles is filled in blue. As the examiner turns the pages, the examinee is required to detect a rule to predict which circle will next be coloured. Moreover, this rule periodically changes without warning. The number of errors made is converted into a scaled score that is interpreted similarly to those of the Hayling.

The Delis-Kaplan Executive Function System: Colour-Word Interference Test (D-KEFS: CWIT).The D-KEFS: CWIT was developed from the Stroop Task and is designed to measure aspects of executive functioning that include the inhibition of learned verbal responses, simultaneous processing, and cognitive flexibility [21]. Four subtests allow for the investigation of these different components [22]: Colour Naming, Word Reading, Inhibition, and Inhibition/Switching. Both the time taken to complete the task and the number of errors made confer performance, which can be converted into age appropriate scaled scores based on stratified normative data. These scaled scores range from 1 to 19 , with a mean of 10 and a standard deviation of 3 , with higher scores indicating better relative performance.

\section{Procedure}

Participants completed the array of tests over several two to three hour sessions. Mr A was also the subject of an unstructured interview regarding his cognition in relation to his daily functioning. The study protocol was approved by the Alfred Hospital Human Research Ethics Committee and participants provided written informed consent prior to their participation.

\section{Data Analysis}

Given that this investigation was a case study, structured quantitative statistical analyses were not possible. Raw scores were converted into appropriate scaled scores, standard scores and percentile ranks based on normative data sets where possible. Differences in performance were noted in terms of the number of standard deviations (1 SD, 2 $\mathrm{SD}$, or $3 \mathrm{SD}$ ) that Mr A's scores were from the mean of the controls (see Rossell et al. [23] for similar approach). In addition, for scores associated with WAIS-IV and WMS-IV, discrepancy analyses for $\mathrm{Mr}$ A were performed.

\section{Results}

\section{Clinical Assessment of Mr A and Controls}

All five participants, including $\mathrm{Mr} \mathrm{A}$, were free from any current psychiatric symptoms and thus Axis 1 disorders. Average scores on the depression, anxiety, and stress subscales of the DASS-21 for the controls were $1.50(\mathrm{SD}=1.00), 0.50(\mathrm{SD}=1.00)$, and $4.75(\mathrm{SD}=$ 3.77), respectively. By contrast, $\mathrm{Mr}$ A obtained a score of 13 on the depression subscale, 6 on the anxiety subscale, and 11 on the stress subscale. Thus, Mr A's subclinical depressive and anxiety symptom scores were greater than three standard deviations from the mean for the controls. Further, his symptoms of stress exceeded the mean for the controls by more than one standard deviation.

\section{Unstructured Interview with Mr A Regarding his Cognitive Functioning}

When asked about any possible cognitive difficulties that might have been experienced, Mr A initially reported that his memory was good. For example, he could remember appointments, dates, addresses, and locations without having to refer to directories, and so seemed to function well. He did, however, state that he relied on various mnemonic devices when trying to learn information, such as attaching information to mental rhythms. When asked whether he found learning information difficult, he expressed that he had to repeat information and that it needed to be associated with something meaningful. He did not seem to believe that he had any noticeable cognitive deficits that would serve to impair his daily functioning.

\section{Neurocognitive Test Results}

The WAIS-IV.As illustrated in Table 1, both Mr A and the controls obtained similarly superior estimates of general intelligence as assessed by the WAIS-IV. As such, Mr A achieved scores on the four cognitive indices that were within or above normative average ranges (i.e. the middle 68\%). Thus, no broad aspect of his cognitive performance could be considered a normative weakness. By contrast, his Perceptual Reasoning index score was strength, being more than two standard deviations greater than the normative mean. A discrepancy analysis (Table 2) revealed that Mr A's Perceptual Reasoning index score was significantly greater than his scores on all of the other cognitive indices. On the other hand, his Working Memory index score was significantly lower than his Verbal Comprehension index score. Similarly, his Perceptual Reasoning index score was more than one standard deviation greater than the average of the controls, whereas his Working Memory index score fell more than two standard deviations below the mean of the controls.

Like his index scores, most of the subtest scaled scores obtained by $\mathrm{Mr}$ A on the WAIS-IV either equalled or exceeded the normative average (Table 1). Notably, however, the subtest scaled scores obtained within the domains of Perceptual Reasoning and Verbal Comprehension differed by five points, indicating non-unitary measures of these domains. Subtest discrepancy analyses (Table 2) showed that Mr A's performance on Digit Span was significantly poorer than his average scaled score of all the subtests; his Digit Span scaled score was more than three standard deviations below the mean of the controls. $\mathrm{Mr}$ A's Digit Span Backwards scaled score of 7 was particularly salient because it was one standard deviation below the normative average and differed by more than two standard deviations from the average of the controls. Despite this, no significant differences were found between the forward, backward, and sequencing components of his Digit Span performance. However, his Digit Span scaled score was significantly poorer than that achieved on Arithmetic.

Of additional note, Mr A's Vocabulary scaled score was significantly worse than his average score on the subtests within the Verbal Comprehension domain scoring below one standard deviation of the average of the controls. By contrast, his performances on Matrix Reasoning and Information were both significantly better than his average subtest score within their respective domains. His Matrix Reasoning scaled score also exceeded one standard deviation of the average of the controls. Finally, Mr A's subtest scatter was 11, which 
Citation: Sumner PJ, Castle DJ, Kaplan RA, Rossell SL (2015) A Neurocognitive Assessment of a Patient with Body Integrity Identity Disorder. Int J Clin Case Stud 1: 107. doi: http://dx.doi.org/10.15344/2455-2356/2015/107

Page 4 of 9

\begin{tabular}{|c|c|c|c|c|c|c|}
\hline & \multicolumn{3}{|l|}{$\mathrm{Mr} \mathrm{A}$} & \multicolumn{3}{|l|}{ Controls } \\
\hline & Raw Score & Scaled Score & Percentile & Average Raw Score & Average Scaled Score & Average Percentile \\
\hline $\begin{array}{l}\text { Full Scale Intelligence } \\
\text { Quotient }\end{array}$ & - & 118.00 & 88.00 & - & $123.00(6.32)$ & $92.25(5.91)$ \\
\hline General Ability Index & - & 127.00 & 96.00 & - & $124(8.37)$ & $92.75(5.06)$ \\
\hline $\begin{array}{l}\text { Verbal } \\
\text { Comprehension } \\
\text { Index }\end{array}$ & - & 114.00 & 82.00 & - & $123.50(13.70)$ & $88.50(13.77)$ \\
\hline $\begin{array}{l}\text { Perceptual Reasoning } \\
\text { Index }\end{array}$ & - & $133.00^{*}$ & 99.00 & - & $118.25(13.60)$ & $82.50(25.01)$ \\
\hline $\begin{array}{l}\text { Working Memory } \\
\text { Index }\end{array}$ & - & $100.00^{\star *}$ & $50.00^{\star * *}$ & - & $116.50(6.35)$ & $85.00(9.24)$ \\
\hline $\begin{array}{l}\text { Processing Speed } \\
\text { Index }\end{array}$ & - & 105.00 & 63.00 & - & $113.25(13.05)$ & $75.00(17.87)$ \\
\hline Vocabulary & $36.00^{*}$ & $10.00^{*}$ & $50.00^{*}$ & $46.25(8.77)$ & $14.00(3.65)$ & $80.65(23.32)$ \\
\hline Information & 21.00 & 15.00 & 95.00 & $21.00(1.41)$ & $14.75(0.96)$ & $93.75(3.40)$ \\
\hline Similarities & 30.00 & 13.00 & 84.00 & $30.50(2.52)$ & $13.50(2.52)$ & $82.50(14.80)$ \\
\hline Block Design & 59.00 & 14.00 & 91.00 & $51.25(15.56)$ & $12.00(3.37)$ & $70.50(36.48)$ \\
\hline Matrix Reasoning & $26.00^{\star}$ & $19.00^{*}$ & $99.90^{*}$ & $23.75(1.50)$ & $15.00(2.00)$ & $93.15(4.30)$ \\
\hline Visual Puzzles & 21.00 & 14.00 & 91.00 & $19.50(4.43)$ & $12.50(3.11)$ & $73.75(32.82)$ \\
\hline Digit Span Total & $25.00^{\star * *}$ & $8.00^{\star * *}$ & $25.00^{* * *}$ & $32.25(1.50)$ & $11.75(0.96)$ & $71.25(10.21)$ \\
\hline Digit Span Forwards & $10.00^{*}$ & $9.00^{*}$ & $37.00^{*}$ & $11.50(1.00)$ & $10.75(1.50)$ & $58.50(17.00)$ \\
\hline Digit Span Backwards & $6.00^{* *}$ & $7.00^{\star *}$ & $16.00^{* *}$ & $10.00(1.41)$ & $11.25(1.71)$ & $64.75(20.40)$ \\
\hline $\begin{array}{ll}\text { Digit } & \text { Span } \\
\text { Sequencing } & \end{array}$ & $9.00^{*}$ & $10.00^{*}$ & $50.00^{*}$ & $10.75(0.96)$ & $12.25(1.50)$ & $75.25(14.43)$ \\
\hline Arithmetic & $17.00^{*}$ & $12^{*}$ & $75.00^{*}$ & $19.00(1.83)$ & $14.25(2.22)$ & $88.25(10.87)$ \\
\hline Coding & 80.00 & 11.00 & 63.00 & $84.00(15.30)$ & $12.25(2.99)$ & $70.50(26.56)$ \\
\hline Symbol Search & 36.00 & 11.00 & 63.00 & $40.00(6.27)$ & $12.75(2.50)$ & $76.75(20.19)$ \\
\hline
\end{tabular}

Table 1: Raw Scores, Scaled Scores and Percentile Ranks Obtained by Mr A and the Controls on the WAIS-IV.

Note: Standard deviations for the controls are displayed in brackets. ${ }^{*}$ Represents values outside of 1 SD from the mean of the controls. ${ }^{* *}$ Represents values outside of 2 SD from the mean of the controls. ${ }^{* * *}$ Represents values outside of $3 \mathrm{SD}$ from the mean of the controls.

occurred in $4.2 \%$ of the normative sample. This scatter was more than one standard deviation greater than the average scatter of the controls $(\mathrm{M}=7.50, \mathrm{SD}=2.38)$.

The WMS-IV.On the WMS-IV, Mr A achieved a Visual Working Memory index score that was within the normative average range, although it was more than three standard deviations lower than the average of the controls (Table 3 ). His performances on the constituent subtests of Visual Working Memory were unremarkable relative to normative data, but his performance on Spatial Addition was more than three standard deviations poorer than the average of the controls. By contrast, Mr A's Logical Memory I and Logical Memory II assessment equalled or exceeded $91 \%$ of the normative sample, and was more than one standard deviation better than the average of the controls. His performance on Visual Reproduction I was of a similar calibre, being equal or better than $84 \%$ of the normative sample. However, his obtained scaled score on Visual Reproduction II was only 8 , more than two standard deviations less than the average of the controls. Likewise, his Visual Reproduction Recognition score was more than three standard deviations below the average of the controls and his score on Logical Memory Recognition was more than one standard deviation less than the average for the controls.
HVLT-R: Mr A's total recall score on the HVLT-R exceeded only $2.90 \%$ of those within the same normative stratum and was more than two standard deviations poorer than the average of the controls (Table 4).

The VOSP: On the whole, Mr A's performance on the VOSP was at ceiling levels (Table 5). A notable exception was his performance on the Silhouettes subtest, which placed him in the $35^{\text {th }}$ percentile for his age. This did not differ from the average of the controls, however.

The D-KEFS: CWIT On the D-KEFS: CWIT, Mr A's performance did not drop below the normative average (Table 6). Notably, the time taken to complete Inhibition was better than average relative to norms, and the total number of errors committed on Inhibition was more than one standard deviation fewer than the average number obtained by the controls. The time taken by $\mathrm{Mr} \mathrm{A}$ to complete the Word Reading component was more than one standard deviation quicker than the average time taken by controls.

The Hayling and Brixton: With regards to the Hayling, Mr A took more than one standard deviation longer than the average for the controls on both sections (Table 7). This translated into an overall scaled score and a scaled score on the first section of the Hayling that were more than one standard deviation lower than the averages that 
Citation: Sumner PJ, Castle DJ, Kaplan RA, Rossell SL (2015) A Neurocognitive Assessment of a Patient with Body Integrity Identity Disorder. Int J Clin Case Stud 1: 107. doi: http://dx.doi.org/10.15344/2455-2356/2015/107

Page 5 of 9

\begin{tabular}{|c|c|c|}
\hline Comparison & Discrepancy & Base Rate \\
\hline \multicolumn{3}{|l|}{ WAIS-IV } \\
\hline \multicolumn{3}{|l|}{ Index Level Analysis } \\
\hline Perceptual Reasoning - Verbal Comprehension & $19.0^{*}$ & 11.0 \\
\hline Perceptual Reasoning - Working Memory & $33.0^{*}$ & 1.5 \\
\hline Perceptual Reasoning - Processing Speed & $22.0^{*}$ & 19.0 \\
\hline Verbal Comprehension - Working Memory & $14.0^{*}$ & 21.5 \\
\hline Verbal Comprehension - Processing Speed & 3.0 & - \\
\hline Processing Speed - Working Memory & 11.0 & - \\
\hline \multicolumn{3}{|l|}{ Subtest Level Analysis } \\
\hline Arithmetic - Digit Span & $4.0^{*}$ & 9.4 \\
\hline Coding - Symbol Search & 2.0 & - \\
\hline Mean Subtest Scaled Score - Block Design\$ & 1.7 & - \\
\hline Similarities - Mean Subtest Scaled Score ${ }^{s}$ & 0.3 & - \\
\hline Mean Subtest Scaled Score - Digit Span & $4.9^{*}$ & $1-2 \%$ \\
\hline Matrix Reasoning - Mean Subtest Scaled Score ${ }^{\$}$ & $3.3^{*}$ & $5-10 \%$ \\
\hline Mean Subtest Scaled Score - Vocabulary ${ }^{s}$ & $2.7^{*}$ & $2-5 \%$ \\
\hline Mean Subtest Scaled Score - Arithmetic & 0.9 & - \\
\hline Mean Subtest Scaled Score - Symbol Search & 1.9 & - \\
\hline Mean Subtest Scaled Score - Visual Puzzles $\$$ & 1.7 & - \\
\hline Information - Mean Subtest Scaled Score & $2.3^{*}$ & $10-15 \%$ \\
\hline Coding - Mean Subtest Scaled Score & 0.1 & - \\
\hline Digit Span Forwards - Digit Span Backwards & 2.0 & - \\
\hline Digit Span Sequencing - Digit Span Forwards & 1.0 & - \\
\hline Digit Span Sequencing - Digit Span Backward & 3.0 & - \\
\hline \multicolumn{3}{|l|}{ WMS-IV } \\
\hline \multicolumn{3}{|l|}{ Subtest Level Analysis } \\
\hline Symbol Span - Spatial Addition & 2.0 & - \\
\hline
\end{tabular}

Table 2: Discrepancy Analyses at the Index and Subtest Levels for Mr A's WAIS-IV and WMS-IV Results.

Note. Ability level was used as the basis for comparison with the WAIS-IV results. * Significant discrepancy $\mathrm{p}<.05$. \$Subtest scaled score was compared to the average scaled score for subtests within its respective cognitive domain.

was influenced by an outlying response on a single item, which, when imputed, resulted in a more typical scaled score for this section and a scaled score of 6 overall. Similarly, one control also obtained a scaled score on section one which was influenced by a slow response to one item, but this did not influence the overall scaled score for this individual. After considering both of these outliers, Mr A's obtained scaled score on the Hayling section one was still more than one standard deviation slower than the average obtained by the controls, although this was no longer the case for his overall scaled score. On the Brixton, the number of errors made by MR A resulted in a scaled score of 10, which placed him in the 99th percentile in relation to norms.

\section{Discussion}

The primary aim of the current study was to investigate patterns of cognition in Mr A. It was hypothesized that Mr A's performance on tests of attention and working memory would be poorer than expected based on age-, education-, and sex-appropriate norms, as well as in comparison to the performance of matched controls. This hypothesis was supported by the data.

One aspect of body integrity identity disorder that had not been previously investigated was whether there are any cognitive components to the disorder. A pattern in Mr A's cognitive abilities emerged in which memory weaknesses were evident. In particular, given $\mathrm{Mr}$ A's high general intelligence and educational level, his performances on tests of working memory were consistently unremarkable with respect to normative data, but were poorer than his performance on tests of other cognitive domains, and were often more than one standard deviation poorer than the performance of the matched controls. Thus, the predicted weakness in Mr A's working memory was supported. Furthermore, his long-term memory seemed weaker than might be predicted from his general intelligence, although this was somewhat less consistent than the results for working memory. Conversely, Mr A was exceptional on tests of visuospatial reasoning. He generally showed superior reasoning, planning, and inhibiting learned responses; functions typically regarded as executive in nature [24,25]. A high level of executive function in conjunction with a pattern of memory weakness was consistent with the daily experiences reported by $\mathrm{Mr} \mathrm{A}$, who claimed to regularly use

\begin{tabular}{|c|c|c|c|c|c|c|}
\hline & $\mathrm{Mr} \mathrm{A}$ & & & Controls & & \\
\hline & Raw Score & Scaled Score & Percentile & Average Raw Score & Average Scaled Score & Average Percentile \\
\hline Visual Working Memory Index & - & $94.00^{* * *}$ & $34.00^{* * *}$ & - & $109.75(5.12)$ & $73.50(11.27)$ \\
\hline Spatial Addition & $12.00^{* * *}$ & $8.00^{* * *}$ & $25.00^{* * *}$ & $19.00(2.16)$ & $12.50(1.29)$ & $78.25(12.09)$ \\
\hline Symbol Span & 25.00 & 10.00 & 50.00 & $28.25(6.13)$ & $10.75(2.63)$ & $57.50(30.99)$ \\
\hline Logical Memory I & $35.00^{*}$ & $14.00^{*}$ & $91.00^{*}$ & $30.50(4.43)$ & $12.25(1.50)$ & $75.25(14.43)$ \\
\hline Logical Memory II & $33.00^{*}$ & $14.00^{*}$ & $91.00^{*}$ & $28.00(4.55)$ & $12.00(1.63)$ & $72.75(16.94)$ \\
\hline Logical Memory Recognition\$ & $24.00^{*}$ & - & $26-50$ & $26.75(2.22)$ & - & - \\
\hline Visual Reproduction I & 42.00 & 13.00 & 84.00 & $40.75(2.06)$ & $11.75(1.71)$ & $69.75(17.46)$ \\
\hline Visual Reproduction II & $23.00^{*}$ & $8.00^{* *}$ & $25.00^{* *}$ & $33.25(6.40)$ & $10.75(1.26)$ & $59.50(16.03)$ \\
\hline
\end{tabular}

Table 3: Raw Scores, Scaled Scores and Percentile Ranks Obtained by Mr A and the Controls on the WMS-IV.

Note: Standard deviations for the controls are displayed in brackets. ${ }^{*}$ Represents values outside of 1 SD from the mean of the controls. ${ }^{* *}$ Represents values outside of 2 SD from the mean of the controls. ${ }^{* *}$ Represents values outside of 3 SD from the mean of the controls. \$Percentiles for Logical Memory Recognition scores are presented as ranges due to highly skewed distributions in the normative sample (Wechsler, 2009b). Thus, the average percentile range of the controls was not calculated.

were obtained by the controls. Moreover, his performance on the first section of the Hayling was at the fifth percentile in relation to norms, whilst his overall score was at the $25^{\text {th }}$ percentile. However, it should be noted that Mr A's performance on the first section of the Hayling mnemonic devices when attempting to learn new information. It appeared possible that his executive abilities allow him to compensate for personal weaknesses in memory.

If one accepts the possibility that Mr A's memory abilities were 
Citation: Sumner PJ, Castle DJ, Kaplan RA, Rossell SL (2015) A Neurocognitive Assessment of a Patient with Body Integrity Identity Disorder. Int J Clin Case Stud 1: 107. doi: http://dx.doi.org/10.15344/2455-2356/2015/107

Page 6 of 9

\begin{tabular}{|l|l|l|l|l|l|l|}
\hline \multicolumn{2}{|l|}{ Mr A } & \multicolumn{2}{l|}{ Controls } \\
\hline & Raw Score & T Score & Percentile & \multicolumn{2}{l|}{$\begin{array}{l}\text { Average Raw } \\
\text { Score }\end{array}$} & \multicolumn{2}{l|}{ Average T Score } & Average Percentile \\
\hline Total Recall & & & $31.00^{* *}$ & $2.90^{*}$ & $28.00(3.61)$ & $48.00(7.21)$ \\
\hline Immediate Recall Trial 1\% & $20.00^{* *}$ & - & 4.98 & $6.67(1.15)$ & - \\
\hline Immediate Recall Trial 2\% & $5.00^{*}$ & $7.00^{* *}$ & - & $2.68^{*}$ & $10.67(1.53)$ & - \\
\hline Immediate Recall Trial 3\% & $8.00^{*}$ & - & $0.78^{*}$ & $10.67(1.53)$ & - \\
\hline Delayed Recall\% & 9.00 & - & 22.21 & $9.33(1.15)$ & - \\
\hline Recognition Discrimination Index & $10.00^{\star * *}$ & - & $\sim 16.00^{* *}$ & $12.00(0.00)$ & - \\
\hline
\end{tabular}

Table 4: Raw Scores, T Scores and Percentile Ranks Obtained by Mr A and the Controls on the HVLT-R.

Note: Standard deviations for the controls are displayed in brackets. ${ }^{*}$ Represents values outside of 1 SD from the mean of the controls. ${ }^{* *}$ Represents values outside of 2 SD from the mean of the controls. ${ }^{* *}$ Represents values outside of $3 \mathrm{SD}$ from the mean of the controls. ${ }^{s}$ Normative data associated with the MCCB is stratified by education in addition to age and gender (Kern et al., 2008). \%Percentiles derived from $\mathrm{Z}$ scores calculated using averages and standard deviations from (age stratified) normative data (Benedict et al.,

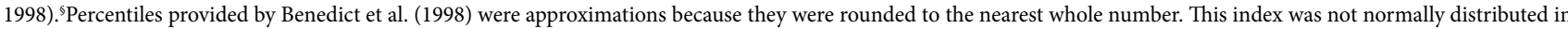
the normative sample; hence precise $\mathrm{Z}$ scores were not calculated.

\begin{tabular}{|c|c|c|c|c|}
\hline & \multicolumn{2}{|r|}{ Mr A } & \multicolumn{2}{|c|}{ Controls } \\
\hline & Raw Score & Percentile & Average Raw Score & Average Percentile \\
\hline Incomplete Letters & 20.00 & 100.00 & $20.00(0.00)$ & $100.00(0.00)$ \\
\hline Silhouettes & 21.00 & 34.90 & $18.25(3.30)$ & $18.90(16.22)$ \\
\hline Object Decision & $20.00^{*}$ & $100.00^{*}$ & $17.75(1.71)$ & $42.73(40.02)$ \\
\hline Progressive Silhouettes & 8.00 & $82.90^{*}$ & $10.75(2.75)$ & $46.45(35.57)$ \\
\hline Dot Counting & 10.00 & 100.00 & $10.00(0.00)$ & $100.00(0.00)$ \\
\hline Position Discrimination & 20.00 & 100.00 & $20.00(0.00)$ & $100.00(0.00)$ \\
\hline Number Location & 10.00 & 100.00 & $10.00(0.00)$ & $100.00(0.00)$ \\
\hline Cube Analysis & 10.00 & 100.00 & $10.00(0.00)$ & $100.00(0.00)$ \\
\hline
\end{tabular}

Table 5: Raw Scores and Percentiles for Mr A and Controls on the VOSP.

Note: Standard deviations for the controls are displayed in brackets. ${ }^{*}$ Represents values outside ofl SD from the mean of the controls.

\begin{tabular}{|l|l|l|l|l|l|l|}
\hline & Mr A & \multicolumn{3}{l|}{ Controls } \\
\hline & Raw Score & Scaled Score & Percentile & Average Raw Score & Average Scaled Score & Average Percentile \\
\hline Colour Naming: Time Taken & 27.93 secs. & 10.00 & 50.00 & 26.25 secs. $(2.63)$ & $10.75(1.50)$ \\
\hline Colour Naming: Total Errors & 0.00 & - & 100 & $0.00(0.00)$ & - \\
\hline Word Reading: Time Taken & 17.81 secs. & $12.00^{*}$ & $75.00^{*}$ & 20.68 secs. (1.83) & $10.75(0.96)$ \\
\hline Word Reading: Total Errors & 0.00 & - & 100 & $0.00(0.00)$ & - & $100.00(0.00)$ \\
\hline Inhibition: Time Taken & 42.06 secs. & 13.00 & 84.00 & 47.03 secs. (10.42) & $11.00(2.16)$ \\
\hline Inhibition: Total Errors & $0.00^{*}$ & $12.00^{*}$ & - & $1.00(0.82)$ & $10.50(12.01)$ \\
\hline Inhibition/Switching: Time Taken & 50.54 secs. & 12.00 & 75.00 & 52.43 secs. (12.89) & $11.25(2.63)$ \\
\hline Inhibition/Switching: Total Errors & 0.00 & 12.00 & - & $0.25(0.50)$ & $11.75(0.50)$ \\
\hline
\end{tabular}

Table 6: Raw Scores Scaled Scores and Percentile Ranks Obtained by Mr A and the Controls on the D-KEFS: CWIT.

Note: Standard deviations for the controls are displayed in brackets. ${ }^{\star}$ Represents values outside of 1 SD from the mean of the controls.

\begin{tabular}{|c|c|c|c|c|c|c|}
\hline & Mr A & & & Controls & & \\
\hline & Raw Score & Scaled Score & Percentile & Raw Score & Scaled Score & Percentile \\
\hline Hayling Time Taken Section 1 & 24.00 secs. $^{*}$ & $3.00^{* *}$ & $5.00^{*}$ & 11.75 secs. $(6.85)$ & $5.25(0.96)$ & $33.75(19.74)$ \\
\hline Hayling Time Taken Section 2 & 35.00 secs. $^{*}$ & 6.00 & 50.00 & 14.75 secs. $(14.97)$ & $6.25(0.50)$ & $56.25(12.50)$ \\
\hline Hayling Total Section 2 Errors & 2.00 & 7.00 & 75.00 & $1.00(1.15)$ & $7.25(0.96)$ & $76.25(18.87)$ \\
\hline Hayling Overall Score & - & $5.00^{*}$ & $25.00^{* *}$ & - & $6.75(0.96)$ & $66.25(19.74)$ \\
\hline Brixton Errors & 7.00 & 10.00 & 99.00 & $10.75(5.56)$ & $8.00(2.31)$ & $74.50(28.29)$ \\
\hline
\end{tabular}

Table 7: Raw Scores, Scaled Scores and Percentile Ranks Obtained by Mr A and the Controls on the Hayling and Brixton.

Note: Standard deviations for the controls are displayed in brackets. ${ }^{*}$ Represents values outside of 1 SD from the mean of the controls. ${ }^{* \star}$ Represents values outside of 2 SD from the mean of the controls. 
Citation: Sumner PJ, Castle DJ, Kaplan RA, Rossell SL (2015) A Neurocognitive Assessment of a Patient with Body Integrity Identity Disorder. Int J Clin Case Stud 1: 107. doi: http://dx.doi.org/10.15344/2455-2356/2015/107

Page 7 of 9

somewhat lower than might be expected given his education and general intelligence, the next question is whether this cognitive pattern is connected to body integrity identity disorder. Demonstrated neurological correlates of body integrity identity disorder include the superior parietal lobule [7,9], a region within a fronto-parietal network that is consistently recruited across tasks of working memory, episodic memory retrieval, conscious visual perception, and attention [10]. However, the precise role of the superior parietal lobule in cognitive tasks is contentious [26]. One idea is that the posterior parietal cortex is specifically involved in multi-modal informational manipulation within working memory. For example, Champod and Petrides [27] utilized a series of visual working memory tasks and found that the right superior parietal lobule was amongst the regions of the posterior parietal cortex specifically implicated when the order of visual stimulus presentation was important for the task as opposed to when the mere presence of particular visual stimulus was important. Further, cogent neuropsychological evidence for the specific role of the superior parietal lobule in manipulation of information in working memory has been produced [28]. Mr A's pattern of results fit with this hypothesis, given his noticeable weaknesses in Spatial Addition and Digit Span Backwards.

Although the right superior parietal lobule was amongst the regions that Champod and Petrides [27] associated with informational manipulation, a replication study using a similar paradigm but with verbal stimuli focused on the role of the intraparietal sulcus[29]. A commonly ascribed function of the intraparietal sulcus is in supporting numeracy and arithmetic [30-32], which Champod and Petrides [27] argued also fit within their informational manipulation hypothesis. However, Mr A's performance on the WAIS-IV arithmetic subtest indicated preserved arithmetic ability. This can be accounted for on two levels. First, the intraparietal sulcus is not amongst the regions of the brain implicated in body integrity identity disorder in terms of changed structure [7] or function [9]. Whilst evidence from fMRI studies do suggest an involvement of the posterior superior parietal lobule in arithmetic [30,31], temporary disruptions to the functioning of this region with Transcranial Magnetic Stimulation have failed to elicit decrements in the performance of mathematical tasks [31]. Thus, the neural region specifically implicated in body integrity identity disorder might not heavily impact performance on arithmetic tests, which is consistent with the current findings.

The second level of perspective for the relative preservation of arithmetic in $\mathrm{Mr} \mathrm{A}$ can be found in light of the literature on embodied cognition, given the research which has specifically investigated numeracy and arithmetic with regards to the representation and use of fingers [30,33-35]. By contrast, Mr A's undesired body part was a portion of his left leg. Therefore, the sensorimotor resources associated with the fingers that are theoretically used to offload cognitive load for arithmetic [35,36] could feasibly be preserved in $\mathrm{Mr} \mathrm{A}$. Incidentally, the neurological investigations into body integrity identity disorder have not included participants with undesired parts of their arm $[7,9]$ It would be an interesting avenue for future research to investigate any potential differences in arithmetic ability between body integrity identity disorder patients with and without undesired body parts that encompass one's fingers.

Berryhill et al. [37] have interpreted the distinction between the manipulation and maintenance of information within an internal attention hypothesis, whereby the posterior parietal cortex is posited to have a role in directing attention towards internal (mnemonic) representations. According to thishypothesis, maintaining information involves continuing attention-based facilitation, which is heavily taxed when manipulation is required because attention must be shifted between original stimuli and updated stimuli. This hypothesis predicts that impaired functioning within the posterior parietal cortex should cause noticeable deficits in tasks requiring manipulation. Crucially however, internal attention has also been used to explain the role of this region in retrieval processes for some aspects of longterm memory [26]. Mr A did exhibit some weakness in both the immediate and delayed free recall of verbal lists, and the delayed free recall of visuospatial information, although this weakness was not apparent in his ability to recognize information or to retrieve more organized information derived from stories. Furthermore, he performed similarly to the matched controls on the WMS-IV Visual Reproduction I. It is notable that lesions within the superior parietal lobule have previously been shown to produce deficits in immediate and delayed visuospatial memory but not in the verbal memory of stories [28].

The neurological overlap between body integrity identity disorder and certain functions of memory can be tentatively contextualized in terms of broader embodied cognition. The relationship between arithmetic and the representation of the fingers has already been alluded to. However, parallels can be drawn from the literature concerning cognition and body representation more broadly. For one, the superior parietal lobule is thought to be involved in the process of integrating information across various modalities that is purportedly important for cognition [10,38], in addition to being central to notions of body representation [39] and body integrity identity disorder [9]. Further, body representation for action has been influenced by motor control theories, which articulate an additional process whereby the sensory and motor consequences of motor commands are simulated [40]. Similarly, advocates of embodied cognition contend that the recruitment of sensorimotor resources for the simulation of external events enables informational offloading, which is important for working memory and long-term memory [36,38,41]. Therefore, the same processes of sensorimotor simulation and integration that occur as part of body representation might also occur as part of cognition. Those with body integrity identity disorder might lack the sensorimotor resources that would otherwise be utilized in difficult cognitive tasks.

It should be noted that $\mathrm{Mr} \mathrm{A}$ also exhibited some substantial cognitive strengths. For example, his ability to reason and plan on tests thought to rely upon frontal functioning was strong. His impressive performance on the visuospatial tasks of the WAIS-IV (and normal functioning on the VOSP) were particularly salient, however, given that the traditional role ascribed to the right posterior parietal cortex is in the processing of visuospatial information (e.g. [42] and, hence, this strength seems somewhat hard to reconcile with the notion of aberrant function within the superior parietal lobule. Nevertheless, lesion-deficit analyses using previous versions of the tests that constitute the Perceptual Reasoning domain suggest that the tasks on which Mr A excelled rely upon inferior parietal, occipital, and superior temporal regions of the right hemisphere [43].Thus, the neural substrate that operates when performing tasks within this domain may be somewhat distinct from those implicated in body integrity identity disorder.

Besides the obvious limitation that the current sample lacked sufficient size necessary for concrete conclusions and generalizations to be made based on statistical comparisons, a main limitation of the present study was that there were no gross brain changes on Mr A's 
Citation: Sumner PJ, Castle DJ, Kaplan RA, Rossell SL (2015) A Neurocognitive Assessment of a Patient with Body Integrity Identity Disorder. Int J Clin Case Stud 1: 107. doi: http://dx.doi.org/10.15344/ijccs/2015/107

Page 8 of 9

structural MRI as reported on by a radiologist. Only two prior studies have shown support for a link between the superior parietal lobule and body integrity identity disorder phenomenology $[7,9]$, one of which included only four subjects with body integrity identity disorder [9]. However, McGeoch and colleagues used functional imaging, Hilti and colleagues detailed region of interest measurements as opposed to the routine clinical report that was available to us. Further theoretical development and enhanced understanding of the neurobiological aspects of body integrity identity disorder, therefore, warrants more detailed empirical investigation with large samples. Comparing behavioural and neurological indices between patients with the disorder and controls whilst undergoing tasks that are reliant on working memory might make for particularly compelling future research.

Another limitation of the current study was the language and cultural differences that existed between $\mathrm{Mr} \mathrm{A}$ and the controls. Namely, Mr A spoke Spanish as his first language, whereas the controls and normative samples were English speaking. Indeed, $\mathrm{Mr}$ A's vocabulary was a personal weakness as assessed by the WAIS-IV, and was less impressive than that of the matched controls. Thus, it is possible that his performances on the cognitive tests were adversely affected by impediments to language. However, several findings suggest that the differences in language contributed minimally to the pattern of results found. First, Mr. A spoke English fluently and articulately, and has been able to successfully attend university within Australia. Second, Mr A's Verbal Comprehension index score was better than his Working Memory index score, and was also similar to the average of the controls. Third, his performance on Arithmetic was better than his performance on Digit Span, the former of which relies more heavily upon language. Finally, the difficulty shown in the various tasks of memory were not limited to the verbal domain. Thus, the patterns of results observed were not interpreted in terms of deficient language skills. Additionally, Mr A scored higher in mood symptoms than the healthy controls. Although some influence of mood on neuropsychological performance cannot be ruled out, $\mathrm{Mr}$ A scores were only in the mild range and are unlikely to have had a significant impact.

Regardless of these limitations, the current study raises the possibility that previous assumptions of preserved cognition in those with body integrity identity disorder were wrong. If consistent cognitive differences can be found at the group level in the future, then current conceptualizations and definitions of body integrity identity disorder may need to be broadened. In addition, such research may add impetus to the search for more effective treatments, given that the best option currently available to those with the disorder appears to lie in them obtaining their desired body modification. However, if the cognitive weaknesses present in $\mathrm{Mr} \mathrm{A}$ can be corroborated, then modifying the body as a treatment option may not address all aspects of the condition.

\section{Conclusion}

In conclusion, Mr A exhibited a pattern of cognitive functioning that was somewhat in line with aberrant functioning within the superior parietal lobule, a region which has been implicated as a neurocorrelate of body integrity identity disorder. In particular, he consistently showed relatively weak performances on tests of working memory.

\section{Competing Interests}

The authors declare that they have no competing interests.

\section{Author Contributions}

All the authors substantially contributed to the study conception and design as well as the acquisition and interpretation of the data and drafting the manuscript.

\section{References}

1. Blanke O, Morgenthaler FD, Brugger P, Overney LS (2009) Preliminary evidence for a fronto-parietal dysfunction in able-bodied participants with a desire for limb amputation. J Neuropsychol 3: 181-200.

2. Blom RM, Hennekam RC, Denys D (2012) Body integrity identity disorder. PLoS One 7: e34702.

3. First MB (2005) Desire for amputation of a limb: paraphilia, psychosis, or a new type of identity disorder. Psychol Med 35: 919-928.

4. First MB, Fisher CE (2012) Body integrity identity disorder: the persistent desire to acquire a physical disability. Psychopathology 45: 3-14.

5. Giummarra MJ, Bradshaw JL, Hilti LM, Nicholls ME, Brugger P (2012) Paralyzed by desire: a new type of body integrity identity disorder. Cogn Behav Neurol 25: 34-41.

6. Bou Khalil R, Richa S (2012) Apotemnophilia or body integrity identity disorder: a case report review. Int J Low Extrem Wounds 11: 313-319.

7. Hilti LM, Hänggi J, Vitacco DA, Kraemer B, Palla A, et al. (2013) The desire for healthy limb amputation: structural brain correlates and clinical features of xenomelia. Brain 136: 318-329.

8. Aoyama A, Krummenacher P, Palla A, Hilti LM, Brugger P, (2012) Impaired spatial-temporal integration of touch in Xenomelia (Body Integrity Identity Disorder). Spatial Cognition \& Computation: An Interdisciplinary Journal 12: 96-110.

9. McGeoch PD, Brang D, Song T, Lee RR, Huang M, et al. (2011) Xenomelia: a new right parietal lobe syndrome. J Neurol Neurosurg Psychiatry 82: 1314-1319.

10. Naghavi HR, Nyberg L (2005) Common fronto-parietal activity in attention, memory, and consciousness: shared demands on integration? Conscious Cogn 14: 390-425.

11. Gaser C, Schlaug G (2003) Brain structures differ between musicians and non-musicians. The Journal of Neuroscience 23: 9240-9245.

12. Oldfield RC (1971) The assessment and analysis of handedness: the Edinburgh inventory. Neuropsychologia 9: 97-113.

13. Sheehan DV, Lecrubier $\mathrm{Y}$, Sheehan $\mathrm{KH}$, Amorim $\mathrm{P}$, Janavs $\mathrm{J}$, et al. (1998) The Mini-International Neuropsychiatric Interview (M.I.N.I.): The development and validation of a structured diagnostic psychiatric interview for DSM-IV and ICD-10. The J Clin Psychiatry 59: 22-33.

14. Lovibond PF, Lovibond SH (1995) The structure of negative emotional states: comparison of the Depression Anxiety Stress Scales (DASS) with the Beck Depression and Anxiety Inventories. Behav Res Ther 33: 335343.

15. Wechsler D (2008) The Wechsler Adult Intelligence Scale - Fourth Edition. San Antonio, Texas: Pearson.

16. Wechsler D (2009a) Wechsler Memory Scale - Fourth Edition. San Antonio, Texas: Pearson.

17. Warrington EK and James M (1991) The Visual Object and Space Perception Battery: Manual. Bury St Edmunds: Thames Valley Test Company.

18. Kern RS, Nuechterlein KH, Green MF, Baade LE, Fenton WS, et al. (2008) The MATRICS Consensus Cognitive Battery, part 2: co-norming and standardization. Am J Psychiatry 165: 214-220.

19. Benedict RHB, Schretlen, D, Groninger L and Brandt J (1998) Hopkins Verbal Learning Test-Revised: Normative data and analysis of inter-form and test-retest reliability. The Clinical Neuropsychologist 12: 43-55. 
Citation: Sumner PJ, Castle DJ, Kaplan RA, Rossell SL (2015) A Neurocognitive Assessment of a Patient with Body Integrity Identity Disorder. Int J Clin Case Stud 1: 107. doi: http://dx.doi.org/10.15344/2455-2356/2015/107

20. Burgess PW, Shallice T (1997) The Hayling and Brixton Tests. London: Pearson Assessment.

21. Swanson J (2005) The Delis-Kaplan Executive Function System: A review. Canadian Journal of School Psychology 20: 117-128.

22. Delis DC, Kaplan E and Kramer JH (2001) Delis Kaplan Executive Function System: Technical Manual. San Antonio, Texas: The Psychological Corporation.

23. Rossell SL, Batty RA, Hughes L (2010) Impaired semantic memory in the formation and maintenance of delusions post-traumatic brain injury: a new cognitive model of delusions. Eur Arch Psychiatry Clin Neurosci 260: 571581.

24. Delis DC, Kaplan E and Kramer JH (2006) Test review. Applied Neuropsychology13: 275-279.

25. White T and Stern RA (2003) Neuropsychological Assessment Battery: Psychometric and Technical Manual. Lutz, Florida: Psychological Assessment Resources, Inc.

26. Olson IR, Berryhill M (2009) Some surprising findings on the involvement of the parietal lobe in human memory. Neurobiol Learn Mem 91: 155-165.

27. Champod AS, Petrides M (2007) Dissociable roles of the posterior parietal and the prefrontal cortex in manipulation and monitoring processes. Proc Natl Acad Sci U S A 104: 14837-14842.

28. Koenigs M, Barbey AK, Postle BR, Grafman J (2009) Superior parietal cortex is critical for the manipulation of information in working memory. J Neurosci 29: 14980-14986.

29. Champod AS and Petrides M (2010) Dissociation within the frontoparietal network in verbal working memory: A parametric functional magnetic resonance imaging study. J Neurosci 30: 3849-3856.

30. Andres M, Michaux N, Pesenti M (2012) Common substrate for mental arithmetic and finger representation in the parietal cortex. Neuroimage 62: 1520-1528.

31. Andres M, Pelgrims B, Michaux N, Olivier E, Pesenti M (2011) Role of distinct parietal areas in arithmetic: an fMRI-guided TMS study. Neuroimage 54: 3048-3056

32. Molko N, Cachia A, Rivière D, Mangin JF, Bruandet M, et al. (2003) Functional and structural alterations of the intraparietal sulcus in a developmental dyscalculia of genetic origin. Neuron 40: 847-858.

33. Andres M, Seron X, Olivier E (2007) Contribution of hand motor circuits to counting. J Cogn Neurosci 19: 563-576.

34. Badets A, Pesenti M, Olivier E (2010) Response-effect compatibility of finger-numeral configurations in arithmetical context. Q J Exp Psychol (Hove) 63: 16-22

35. Krause F, Bekkering $\mathrm{H}$, Lindemann $\mathrm{O}$ (2013) A feeling for numbers: shared metric for symbolic and tactile numerosities. Front Psychol 4: 7.

36. Wilson M (2002) Six views of embodied cognition. Psychonomic Bulletin \& Review 9: 625-636.

37. Berryhill ME, Chein J, Olson IR (2011) At the intersection of attention and memory: the mechanistic role of the posterior parietal lobe in working memory. Neuropsychologia 49: 1306-1315.

38. Barsalou LW (2008) Grounded cognition. Annu Rev Psychol 59: 617-645.

39. de Vignemont F (2010) Body schema and body image--pros and cons. Neuropsychologia 48: 669-680.

40. Wolpert DM, Ghahramani Z and Jordan MI (1995) An internal model for sensorimotor integration. Science 269: 1880-1882.

41. Wilson M (2001) The case for sensorimotor coding in working memory. Psychon Bull Rev 8: 44-57.

42. Russell C, Deidda C, Malhotra P, Crinion JT, Merola S, et al. (2010) A deficit of spatial remapping in constructional apraxia after right-hemisphere stroke. Brain 133: 1239-1251.

43. Gläscher J, Tranel D, Paul LK, Rudrauf D, Rorden C, et al. (2009) Lesion mapping of cognitive abilities linked to intelligence. Neuron 61: 681-691. 